\title{
Representational Account of Memory: Insights from Aging and Synesthesia
}

\author{
Gaby Pfeifer ${ }^{1}$, Jamie Ward ${ }^{1}$, Dennis Chan ${ }^{2}$, and Natasha Sigala ${ }^{1}$
}

\begin{abstract}
The representational account of memory envisages perception and memory to be on a continuum rather than in discretely divided brain systems [Bussey, T. J., \& Saksida, L. M. Memory, perception, and the ventral visual-perirhinal-hippocampal stream: Thinking outside of the boxes. Hippocampus, 17, 898-908, 2007]. We tested this account using a novel between-group design with young grapheme-color synesthetes, older adults, and young controls. We investigated how the disparate sensoryperceptual abilities between these groups translated into associative memory performance for visual stimuli that do not induce synesthesia. ROI analyses of the entire ventral visual stream showed that associative retrieval (a pair-associate retrieved in the absence of a visual stimulus) yielded enhanced activity in young and older adults' visual regions relative to synesthetes, whereas associative recognition (deciding whether a visual stim-
\end{abstract}

\section{INTRODUCTION}

The notion that perception and memory share similar neural representations is well established (Buchsbaum, Lemire-Rodger, Fang, \& Abdi, 2012; Bussey \& Saksida, 2007; Wheeler, Petersen, \& Buckner, 2000). In keeping with this principle, findings from the literature on normal aging show that reduced perception (McDonough, Cervantes, Gray, \& Gallo, 2014) and declining sensory functions (Humes, Busey, Craig, \& Kewley-Port, 2013) are associated with poorer memory performance in older adults (McDonough et al., 2014). Age-related reductions in early visual cortex sensitivity (Wang et al., 2014) and a neural dedifferentiation in ventral visual cortex, leading to loss of neuronal sensitivity and specificity for visual stimuli (Park et al., 2004, 2012), can account for problems in visual perception and episodic retrieval (Park \& McDonough, 2013; Goh, 2011). However, the causal influence of perception on memory is still unclear (St-Laurent, Abdi, Bondad, \& Buchsbaum, 2014). For instance, older adults typically compensate degraded sensory-perceptual functions with enhanced activity in $\mathrm{pFC}$, described as the posterior-to-anterior shift in aging (Davis, Dennis, Daselaar, Fleck, \& Cabeza, 2008). But do enhanced sensory-perceptual functions correlate with

\footnotetext{
${ }^{1}$ University of Sussex, ${ }^{2}$ University of Cambridge
}

ulus was the correct pair-associate) was characterized by enhanced activity in synesthetes' visual regions relative to older adults. Whole-brain analyses at associative retrieval revealed an effect of age in early visual cortex, with older adults showing enhanced activity relative to synesthetes and young adults. At associative recognition, the group effect was reversed: Synesthetes showed significantly enhanced activity relative to young and older adults in early visual regions. The inverted group effects observed between retrieval and recognition indicate that reduced sensitivity in visual cortex (as in aging) comes with increased activity during top-down retrieval and decreased activity during bottom-up recognition, whereas enhanced sensitivity (as in synesthesia) shows the opposite pattern. Our results provide novel evidence for the direct contribution of perceptual mechanisms to visual associative memory based on the examples of synesthesia and aging.

reduced pFC activity and a more efficient retrieval system? This can be addressed by studying grapheme-color synesthetes, whose enhanced perceptual mechanisms result in the perception of monochrome letters, words, or digits as colored (e.g., Ward, 2013). Synesthetes also show enhanced early visual cortex sensitivity to non-synesthesiainducing stimuli (Barnett et al., 2008) and greater excitability of visual cortex relative to controls (Terhune, Tai, Cowey, Popescu, \& Kadosh, 2011). An increasing number of studies further reveal that synesthetes have a memory advantage for verbal and visual stimuli (Rothen, Meier, \& Ward, 2012). Specifically, the memory advantage is found irrespective of whether stimuli evoke synesthetic colors (Radvansky, Gibson, \& McNerney, 2011; Rothen \& Meier, 2010), contain real colors (Pritchard, Rothen, Coolbear, \& Ward, 2013; Yaro \& Ward, 2007), or are abstract, achromatic in nature and hence do not elicit synesthesia (Pfeifer, Rothen, Ward, Chan, \& Sigala, 2014; Ward, Hovard, Jones, \& Rothen, 2013; Gross, Neargarder, Caldwell-Harris, \& Cronin-Golomb, 2011; Rothen \& Meier, 2010). The latter finding suggests that synesthetes might be endowed with a generic memory advantage for visual stimuli, spurred by their enhanced sensitivity in visual cortex. Here, we investigated how the disparate sensory and perceptual abilities of three participant groups (young grapheme-color synesthetes, young adults, and older adults) translate into visual associative memory for non-synesthesia-inducing, 
achromatic abstract pair-associates. The scientific rationale for studying perception and memory in older adults and synesthetes is based on the principles of the representational account of memory (Bussey \& Saksida, 2007). According to this account, visual stimuli are represented as a perceptual-mnemonic continuum along the ventral visual stream (VVS). Simple features are processed in early, less-selective primary visual regions and become further unitized and processed as complex features in rostral temporal regions, including the perirhinal cortex (PRC) and the hippocampus. There is no anatomical divide of memory and perception in respective medial-temporal lobe structures and sensory cortices. Instead, memory retrieval is envisaged as a stimulus-dependent hierarchical process that takes place in dedicated brain structures along the VVS. These principles are in opposition to modular views of memory that postulate a specific role for the hippocampus and neighboring medial-temporal lobe structures in declarative memory and a role for posterior ventral visual regions for visual perception (Eichenbaum, 2013; Squire \& Wixted, 2011; Yonelinas, Aly, Wang, \& Koen, 2010). The model is well suited to explain the unusual color perceptions in synesthetes, which presumably emerge from a cross-wiring of the letter and color area in posterior VVS (Hubbard, Brang, \& Ramachandran, 2011; Ramachandran \& Hubbard, 2001). This model proposes that the same neural substrates that underpin the synesthetes' color perceptions will give rise to a memory advantage for verbal and color stimuli (Rothen et al., 2012). Likewise, the model predicts that synesthetes will have a generic memory advantage for achromatic, nonsynesthesia-inducing stimuli (Pfeifer et al., 2014; Gross et al., 2011; Rothen \& Meier, 2010), as a result of enhanced sensory processing in early visual cortex (Terhune et al., 2011; Barnett et al., 2008). Taken together, the synesthetes' enhanced sensation and perception in response to visual stimuli is underpinned by neural substrates that are likely to boost visual memory as well. With respect to aging, cognitive theories such as the differentiation account (Park \& McDonough, 2013) further converge with the representational account, suggesting a link between sensoryperceptual functions and higher cognitive functioning. For example, Park, Carp, Hebrank, Park, and Polk (2010) showed that the neural specificity in ventral visual areas predicted task performance on measures of fluid intelligence (digit symbol, dot comparison, trail-making, and verbal fluency tasks). Specifically, correlations between neural specificity and behavioral performance on these measures were significant after controlling for age, suggesting a generic link between sensory and cognitive functioning. It is important to note that age-related perceptual deficits are not all underpinned by the same neural changes across the brain. Park et al. (2012) found that, in response to processing faces, the extended face network (amygdala, inferior frontal gyrus, orbitofrontal cortex) showed reduced activity in older versus young adults. By contrast, the core face network (fusiform gyrus, occipital face area, and superior temporal sulcus) was characterized by age-related neural broadening, whereby neurons had lost their sensitivity and responded to faces and houses with comparable strength (see also Park et al., 2004). This study demonstrates that posterior, sensory regions might be particularly prone to reduced neural sensitivity in older adults, which has further shown to affect visual memory: Kalkstein, Checksfield, Bollinger, and Gazzaley (2011) used a visual recognition test and found that older adults with poorer, but not those with comparable, memory performance relative to young adults showed a neural dedifferentiation in the visual motion processing area V5 during mental imagery of moving dots. These results support and extend the link between sensory and cognitive functioning, showing that the fidelity of sensory regions in visual cortex predicts neural sensitivity of imagined stimuli and is associated with enhanced visual memory. In the present fMRI study, we focused on the representational account of memory as a neural model to explain the link between sensory-perceptual functions and memory performance. The advantage of this model is its prediction of stimulus representations in specific ventral temporal regions that subserve perception and memory alike. To date, the model has mainly received support from anterior regions of the VVS, including the PRC and hippocampus (see Saksida \& Bussey, 2010, for a comprehensive review). However, given the known differences between synesthetes and older adults in sensory association cortex, we expected to extend the evidence for the model to posterior regions of the VVS. We employed a delayed pair-associative (DPA) retrieval task and conducted ROI and whole-brain analyses for two types of memory: associative retrieval and recognition. For our ROI analysis, we reasoned that if synesthetes were more efficient at associative retrieval (as a result of enhanced sensory processing in early visual regions; Barnett et al., 2008), this would manifest as reduced activation relative to the other two groups, especially in posterior regions of the VVS. At associative recognition, the synesthetes were predicted to differ from young and older adults in one of two ways: The synesthetes' hyperexcitability in response to bottom-up perceptual targets (Terhune et al., 2011) could manifest as heightened activity in early visual regions of the VVS. Likewise, we might find reduced activation in some areas of the synesthetes' VVS, where few but dedicated neurons code for specific classes of visual stimuli. These areas would be represented by weaker BOLD signal in fMRI (e.g., Kok, Jehee, \& de Lange, 2012) and consequently appear as reduced activation. For our whole-brain analyses, we hypothesized that young synesthetes would show the most differentiated activation pattern during associative retrieval and recognition. Specifically, such a differentiated activation pattern would be characterized by fewer but dedicated brain areas in posterior occipitotemporal regions relative to young and/or older adults, whereas older adults would show more pronounced activation in $\mathrm{pFC}$ as a result of posterior-to-anterior shift in aging (Davis et al., 2008). 


\section{METHODS}

\section{Participants}

Nineteen young adults (eight women; age range $=21-$ 32 years; $M=24.32$ years), 19 older adults (11 women; age range $=59-81$ years; $M=66.21$ years $)$, and 19 young grapheme-color synesthetes (15 women; age range = 19-33 years; $M=23.00$ years) took part in the experiment after giving their informed, written consent. The study was reviewed and approved by the Brighton and Sussex Medical School research governance and ethics committee, which follows the guidelines of the World Medical Association Declaration of Helsinki. The participants had no history of psychiatric or neurological diseases. The average number of years of formal education was $M=16.95$ (SD = 1.68) for young adults, $M=13.95$ $(S D=3.32)$ for older adults, and $M=16.74(S D=2.11)$ for the synesthetes. The groups differed in the number of years of education, $F(2,54)=8.717, p=.001$. Tukey post hoc comparisons showed that the difference was significant between young and older adults $(p=.001)$, between synesthetes and older adults $(p=.003)$, but not between young adults and synesthetes $(p=.963)$. Screening for cognitive impairment was carried out for all but five young adults, using the Mini-Mental State Examination (Folstein, Folstein, \& McHugh, 1975). All participants performed comparably on the Mini-Mental State Examination, $F(2,51)=2.11, p=.131$, with high average scores across the 14 young adults $(M=28.93$, $S D=0.93), 19$ older adults $(M=28.15, S D=1.46)$, and 19 synesthetes $(M=28.89, S D=1.37)$. Synesthetes were recruited from the University of Sussex and via the UK Synaesthesia Association website www.uksynaesthesia. com. All synesthetes reported seeing colors in response to letters or digits. To verify synesthesia, we used the "Synesthesia battery" (Eagleman, Kagan, Nelson, Sagaram, \& Sarma, 2007), available on www.synesthete.org, and the adapted a cutoff score of 1.43 (Rothen, Seth, Witzel, \& Ward, 2013). Using this battery, a mean score of $M=$ $0.81(S D=0.28$; range $=0.38-1.39)$ was obtained across our group of synesthetes, which is consistent with synesthesia.

\section{Experimental Design and Stimuli}

The fMRI protocol consisted of a DPA task (experimental condition) and a delayed matching-to-sample (DMS) task (control condition). The tasks were administered in separate scanning sessions, with the DPA task always presented first to avoid retroactive interference effects on associative memory.

\section{DPA task}

For the DPA task, we selected eight pair-associates (black-and-white fractal images) from a pool of 16 pairs that were rated for visual similarity by an independent group of 20 participants. Participants gave their ratings on a 5-point Likert scale (Likert, 1932), where a rating of 1 indicated no visual similarity and a rating of 5 indicated high visual similarity between pairs. On the basis of the mean ratings, we selected the four most dissimilar and the four most similar pairs, respectively, representing high- and low-memory load conditions (example pairs illustrated in Figure 1). A Wilcoxon signed-rank test demonstrated that the four selected similar pairs were rated significantly higher in visual similarity $(M=$ $3.87, S D=0.38)$ compared with the four selected dissimilar pairs $(M=1.31, S D=0.20)$; significance $Z=$ $-2.521, p=.012$ (two-tailed). We used an event-related design, during which each of the selected pairs was randomly presented eight times, amounting to a total of 32 similar and 32 dissimilar pairs. The cue and target images were presented interchangeably throughout the task. On $62.5 \%$ of the trials, the cue pictures were followed by a matching target, constituting 40 match trials and 24 nonmatch trials. In this sense, lure stimuli were nonmatching images from the same set of the eight pair-associates rather than trial unique stimuli. Using recombinations of same-set stimuli constitutes a more powerful test of associative memory, requiring participants to retrieve the intact combination of pairassociates out of equally familiar stimuli rather than rejecting lures on the basis of their novelty (Mayes, Montaldi, \& Migo, 2007).
Figure 1. Example stimuli of the four visually similar and four visually dissimilar pairs used in the DPA task.

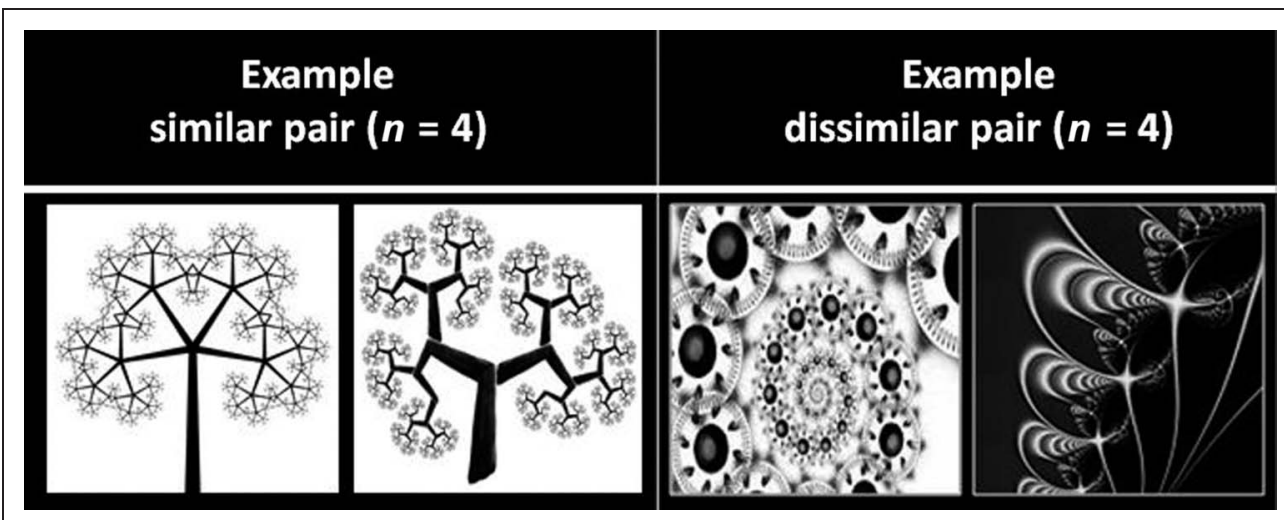




\section{DMS task}

For the DMS task, we chose an independent set of eight individual black-and-white fractal images. We used an event-related design, consisting of a pseudorandom presentation of 32 individual fractal images, with each of the selected eight images shown four times. On $62.5 \%$ of the trials, the cue pictures were followed by a matching target, constituting 20 match trials and 12 nonmatch trials. Like in the DPA task, lure stimuli were nonmatching images from the same set of the eight fractals rather than trial unique stimuli. Across the DPA and DMS tasks, the minimum trial distance between match and nonmatch trials was 1 (i.e., a match trial could immediately follow a nonmatch trial and vice versa), and the maximum trial distance was 5 (i.e., a nonmatch trial could follow four presentations of match trials).

\section{Training and Scanning Procedure}

\section{Associative Learning Task}

Before scanning, participants were trained on the fractal pair-associates of the DPA task. They were explicitly informed that they would be given a memory test on these stimuli during scanning. Each of the eight pairassociates was randomly presented once at the center of a computer screen for $4 \mathrm{sec}$, and participants were instructed to remember the correct association of the pairs. The presentation was followed by a trial-anderror learning task. In this task, each trial began with a fixation cross presented for $2 \mathrm{sec}$, followed by a cue picture presented at the top of the screen and four possible matching target pictures below (Figure 2A). The targets were taken from the stimulus set of the eight pair-associates, and one target was always a match. Participants were asked to indicate which of the four targets belonged with the cue by using different keyboard responses for each target. The pictures stayed on screen until a response was recorded. Following the response, visual feedback appeared below the pictures for $3 \mathrm{sec}$, indicating whether or not the matching target had been identified correctly (green tick or red cross respectively). Each run contained eight trials, and participants performed the test until they achieved a minimum of seven of eight hits on two successive runs (learning criterion). A minimum of two runs was required in the learning phase. Cue and target shapes of all pair-associates were presented interchangeably during learning: a stimulus that had been presented as the cue in one run constituted the target in the following run. Stimuli were delivered using Presentation 14.9 (Neurobiobehavioral Systems, Inc., Berkeley, CA).

\section{DPA and DMS tasks}

Following the associative learning task, participants were familiarized with the DPA and DMS tasks before scan- ning. During scanning, an identical trial structure was used across the DPA and DMS tasks (Figure 2B). During the cue period $(1 \mathrm{sec})$ of the DPA task, participants were asked to use the cue to retrieve the matching target (associative retrieval). During the cue period ( $1 \mathrm{sec}$ ) of the DMS task, participants were asked to build up a mental image of the cue. The delay period $(8 \mathrm{sec})$ required participants to either hold the retrieved picture in mind (DPA task) or to hold the cue image in mind (DMS task). Finally, the target presentation (1 sec) in the DPA task comprised the associative recognition stage, where participants were asked to recognize the target as the matching or nonmatching pair-associate. The target presentation $(1 \mathrm{sec})$ of the DMS task required participants to judge whether the target was the identical image to the cue. Following target presentation in both tasks, a response window appeared and stayed on screen for $5 \mathrm{sec}$, during which participants were asked to press one of four buttons, providing combined decisions about the target (match/nonmatch) and self-rated confidence (confident/not sure). The button-presses were followed by a variable intertrial interval of $6-12 \mathrm{sec}$ before the next trial (Figure 2B).

\section{Data Acquisition}

Imaging data were collected using a Siemens Magnetom Avanto 1.5-T MRI scanner (Siemens, Erlangen, Germany) with a 32-channel phased-array head coil, tuned to 66.6 MHz. Visual stimuli were presented on an in-bore rear projection screen, at a viewing distance of approximately $45 \mathrm{~cm}$, subtending $5^{\circ}$ of visual angle. Stimuli were delivered using Cogent2000 v1.32 running under MATLAB R2006b (The MathWorks, Inc., Natick, MA). Time-course series of the two runs were acquired using a T2*-weighted EPI sequence, obtaining 644 volumes during the DPA task and 324 volumes during the DMS task. Each volume consisted of 35 axial slices oriented in parallel to the AC-PC line and covering the whole brain. Slices were acquired bottom-up in the interleaved mode. The following functional imaging parameters were used: repetition time $(\mathrm{TR})=2620 \mathrm{msec}$, echo time $(\mathrm{TE})=42 \mathrm{msec}$, flip angle $=90^{\circ}$, matrix $=64 \times$ 64 , field of view $=192 \times 192 \mathrm{~mm}$, slice thickness $=3.0 \mathrm{~mm}$ with a $20 \%$ gap, resulting in $3.0 \mathrm{~mm}$ isotropic voxels. To aid distortion correction, corresponding phase and magnitude field maps were acquired with the following: TR $=$ $513 \mathrm{msec}$, TE $1=5.78 \mathrm{msec}$, TE2 $=10.54 \mathrm{msec}$, flip angle $=$ $60^{\circ}$. A whole-brain, high-resolution T1-weighted 3-D structural image was obtained using a magnetization-prepared gradient-echo sequence, consisting of 192 contiguous axial slices $(\mathrm{TR}=1160 \mathrm{msec}, \mathrm{TE}=4.24 \mathrm{msec}$, flip angle $=$ $15^{\circ}$, matrix $=256 \times 256$, field of view $=230 \times 230 \mathrm{~mm}$, $0.9 \mathrm{~mm}$ isotropic voxel size). The T1-weighted image was used as an anatomical reference for each participant's functional data. 


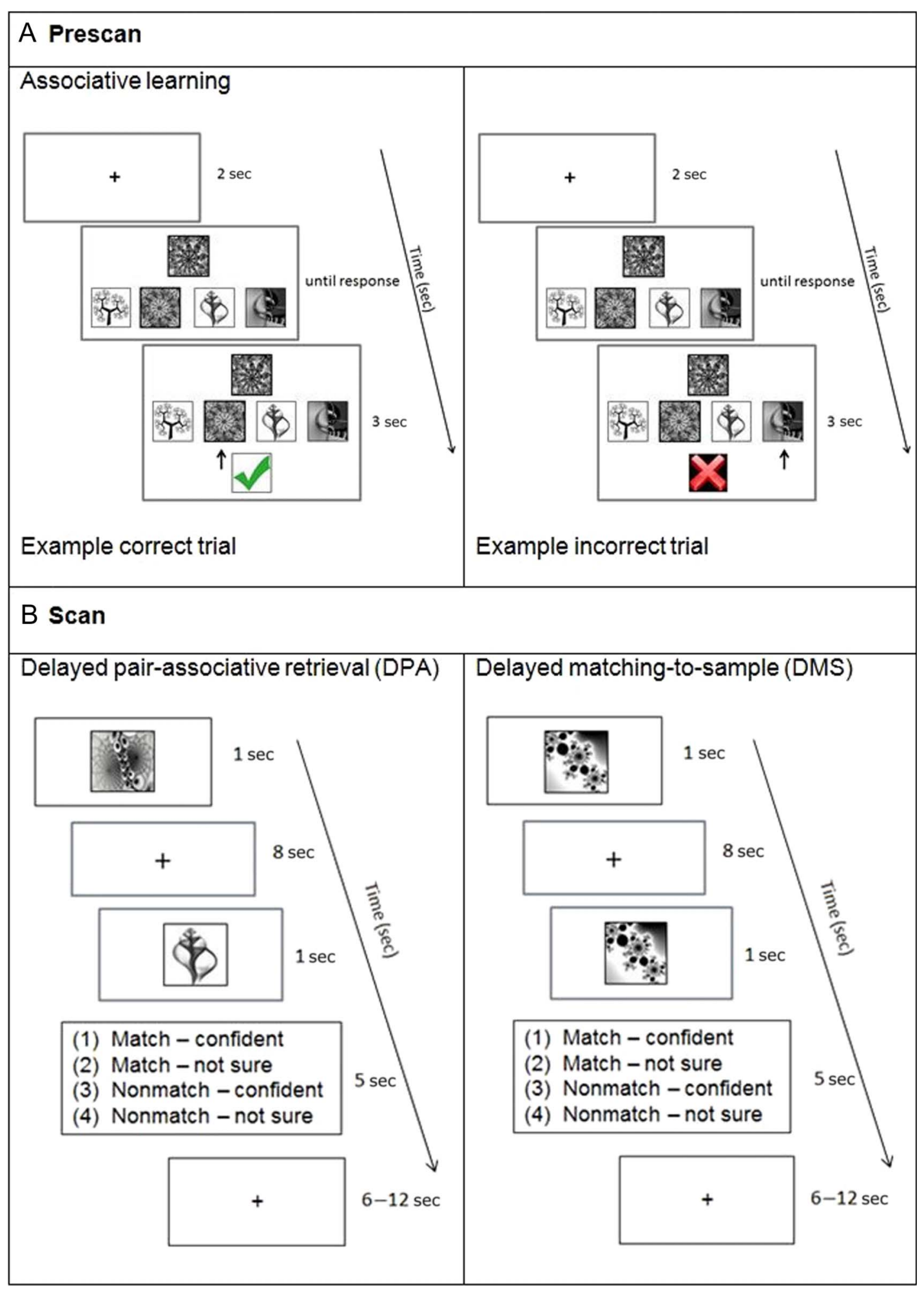

Figure 2. Experimental design. (A) The prescan phase involved a four alternative forced-choice trial-and-error learning task of eight pair-associates. Panels from top to bottom: Fixation cross; stimulus presentation during which participants were asked to select one of four possible pair-associates from the bottom of the screen to match the cue image at the top; stimulus presentation plus visual feedback. Example trials of a correct (left) and incorrect (right) response are shown. (B) The scan phase involved two types of trials, DPA and DMS. DPA trials required participants to retrieve a cue's matching pair-associate and hold it in mind over an 8-sec delay. DMS trials required participants to hold the cue in mind over an 8-sec delay. Upon target presentation, participants were asked to decide whether the target was a match or nonmatch (in DPA and DMS trials) and give their responses within a 5-sec time window. 


\section{fMRI Analyses}

We used SPM8 (Wellcome Trust Centre for Neuroimaging, UCL, London, UK; www.fil.ion.ucl.ac.uk $\backslash \mathrm{spm}$ ) running under MATLAB R2013a for data preprocessing and statistical analyses. Preprocessing of functional images was carried out for each task separately, including slicetime correction to the middle slice in time, spatial realignment to the first image, and unwarping using the acquired field maps. The T1-weighted structural image was coregistered to the mean functional image and subsequently segmented to obtain normalization parameters based on the standard Montreal Neurological Institute (MNI) template. The segmentation parameters were used to transform each subject's functional images and the bias-corrected structural image into MNI space. Voxel sizes of the functional and structural images were retained during normalization, and the normalized functional images were spatially smoothed using an 8-mm Gaussian kernel (FWHM). Statistical analyses were performed using the general linear model. At the singlesubject analysis, the DPA and DMS tasks were entered as separate sessions into the model. For the DPA task, we separated the similar and dissimilar trials and specified regressors associated with the cue and target period for each condition. The target period was further separated into match and nonmatch trials. This resulted in two regressors of interest relating to associative retrieval (similar_cue $[\mathrm{sC}]$, dissimilar_cue $[\mathrm{dC}]$ ) and four regressors of interest relating to associative recognition (similar_target_match [sTM], similar_target_nonmatch [sTNM], dissimilar_target_match [dTM], dissimilar_target nonmatch $[\mathrm{dTNM}]$ ). (An additional task-related regressor was specified for the delay period, the results of which are not reported in this study). Associative retrieval (cue period) was analyzed by including only accurate and confident responses in the $\mathrm{sC}$ or $\mathrm{dC}$ regressors (collapsing across confident hit and correct rejection trials). Associative recognition (target period) was analyzed by including confident hits in the STM or dTM regressors and confident correct rejections in the sTNM or dTNM regressors, respectively. For all target regressors, we used an equal number of match and nonmatch trials for each participant. Match and nonmatch trials were equated by randomly excluding surplus trials of either trial type. Regressors of no interest included the prompt (containing participant's button presses), a nuisance regressor (containing all misses, false alarms, nonconfident responses, nonresponses), and six regressors representing motionrelated variance. For the DMS task, we used the identical regressor specification as for the DPA task (modeling cue, delay, and target periods), with only one condition. The main regressors of interest were the DMS-related activity of the cue and target period (DMS cue [DMSC] and DMS target [DMST]), containing accurate and confident responses (collapsed across confident hit and correct rejection trials). These regressors served as a control condition for DPA-related activity of the cue and target period to account for perceptual and working memoryrelated signals and retain activity related to associative retrieval and recognition (Ranganath, Cohen, Dam, \& D'Esposito, 2004). Regressors of no interest for the DMS task included one prompt, one nuisance, and six motion regressors (as above). Modeling of regressors of interest was identical across the DPA and DMS tasks, given the identical trial structure: For each regressor representing a cue and target period, activation was modeled using a boxcar function starting at onset and lasting for $1 \mathrm{sec}$. All regressors were convolved with a canonical hemodynamic response function available in SPM8 (Friston et al., 1998). A high-pass filter was applied with a period of $128 \mathrm{sec}$ to remove low-frequency signals relating to scanner drift and/or physiological noise.

\section{Gray Matter Volume Analyses}

Given that we compared a group of 19 older adults against 38 younger adults (19 synesthetes and 19 controls) and had an unequal sex distribution across our 57 participants (male: $n=23$; female: $n=34$ ), we calculated each participants' total and regional gray matter (GM) volume (in $\mathrm{ml}$ ), which was subsequently entered as a covariate in all second-level fMRI analyses to indirectly account for age- (Lemaitre et al., 2005; Raz et al., 2005) and sex-related (Luders, Steinmetz, \& Jancke, 2002) GM volume differences. Total GM volume was calculated from the subject-specific GM masks in native space, which were obtained following the segmentation of participants' highresolution structural T1 images. Participants' regional GM volume was extracted from 12 anatomically defined ROIs (see ROI Analyses) and served as ROI-specific covariates in our ROI analyses. Given that the masks of the anatomical ROIs were in standard (MNI) space, regional GM volume within these ROIs was extracted from participants' normalized brains. This involved the spatial normalization of each participant's structural T1 image to MNI space using the preprocessing normalization parameters. Total GM volume was segmented from these normalized structural T1 images. All segmented, normalized GM images underwent a Jacobian modulation. No smoothing was applied for the purpose of avoiding GM overlap from neighboring brain regions.

\section{Second-level Analyses}

Results of the single-subject analyses were taken to group level by computing several ANOVAs for the cue (associative retrieval) and target periods (associative recognition). For the cue period, two independent oneway ANOVAs were computed with Group (young adults, older adults, synesthetes) as the between-subject factor, for which the respective contrast images of the similar and dissimilar condition relative the control task were used ( $\mathrm{sC}>$ DMSC and $\mathrm{dC}>$ DMSC, respectively). We computed the average task activity from the contrasts 
$(\mathrm{sC}>\mathrm{DMSC})$ and $(\mathrm{dC}>\mathrm{DMSC})$ using a $t$-contrast across groups for each ANOVA. Exclusive masks of the average task activity were saved for analysis of group effects. All parametric maps and masks derived from the above ANOVAs were thresholded at $p<.001$ (uncorrected), with an extent threshold of $k=5$ voxels (following the significance levels set for experiments with comparably rigorous control tasks, e.g., Staresina \& Davachi, 2010; Schott et al., 2005; Ranganath et al., 2004). To investigate group differences, we computed two independent oneway ANOVAs by entering the beta images $\mathrm{sC}$ and $\mathrm{dC}$. Using $F$-contrasts, the group effects of $\mathrm{sC}$ and $\mathrm{dC}$ were inclusively masked with the average task activity and suprathresholded at $p<.001$ (uncorrected), $k=5$ voxels. Thus, the masking served three purposes: (1) it constrained the signal to areas for which the effects of the control task were subtracted; (2) it ensured that we reported group differences within task-related regions that show significant activations above zero; and (3) it increased the threshold of identified voxels, which had to survive the $p<.001$ (uncorrected), $k=5$ voxels threshold of the task activity and the group effect (Daselaar, Porat, Huijbers, \& Pennartz, 2010). For the target period, the subject-specific beta images of similar and dissimilar match and nonmatch trials (sTM, dTM, sTNM, and dTNM) were subjected to a $3 \times 2 \times 2$ mixed ANOVA using the full factorial design specification in SPM8. Group (young adults, older adults, synesthetes) was entered as the between-subject factor, and Condition (similar and dissimilar pair-associates) and Target type (match and nonmatches) as the within-subject factors. All main and interaction effects derived from the ANOVA are reported at a threshold of $p<.05$ (FWE-corrected), with an extent threshold of $k=5$ voxels. To examine activation differences between associative recognition (DPA task) and recognition of images that had to be held in working memory (DMS task), we further computed two independent one-way ANOVAs, entering Group as the between-subject factor and the contrast images similar_target $>$ DMS_target $(\mathrm{sT}>$ DMST) and dissimilar_target $>$ DMS_Target $(\mathrm{dT}>$ DMST), respectively. Using this analysis, we averaged across trial type (match, nonmatch) to increase the signal and account for the more stringent comparison against the control task. The results of these two ANOVAs were reported at a threshold of $p<.001, k=5$ voxels.

\section{ROI Analyses}

These were carried out for associative retrieval and recognition to specifically test the representational account of memory by mapping out the entire ventral visual stream. To this end, we specified six anatomical ROIs bilaterally: inferior occipital gyrus, posterior inferior temporal gyrus, anterior inferior temporal gyrus, fusiform gyrus, PRC, and the hippocampus. The mask for the PRC was taken from Holdstock, Hocking, Notley, Devlin, and Price (2009), available on www.neurolang.com/ research/perirhinal-map/. The hippocampus was taken from the Anatomy toolbox v1.8, 2011 (Eickhoff et al., 2005), containing the substructures subiculum, cornu ammonis, dentate gyrus, and the hippocampal-amygdala transition area. Because this mask extended into neighboring regions including the entorhinal cortex, thalamus, and the ventricles, we manually retraced it for both hemispheres to exclude these areas. For the drawing, the mask of the Anatomy toolbox was overlaid on the single-subject brain in MRIcron as a guide to ensure that all relevant substructures were retained. All other masks were from the WFU PickAtlas v2.4 (www.nitrc. org/projects/wfu_pickatlas/; Maldjian, Laurienti, Kraft, \& Burdette, 2003).

For the ROI analyses, we used nonsmoothed images to reduce signal overlap from neighboring brain areas. For simplicity, the beta images for the cue period (associative retrieval) and target period (associative recognition) were averaged across condition (similar, dissimilar) and target type (match, nonmatch). For each ROI, separate one-way ANOVAs were computed in SPM, with Group (young adults, older adults, synesthetes) as the betweensubject factor and the respective beta images for the cue and target period as the within-subject factor. Each ANOVA model contained the participants' ROI-specific GM volume to account for age and individual differences. For each model, we computed the average signal in each ROI across groups (i.e., the average task activity) and applied a threshold of $p<.005$ (uncorrected), $k=0$ voxels. Using the rfxplot toolbox (Gläscher, 2009) available in SPM8, we then extracted the percent signal change for each group. The percent signal change was then subjected to three separate $3 \times 6$ mixed ANOVAs (i.e., associative retrieval [DPA cue period], working memory control [DMS cue period], associative recognition [DPA target period], and working memory recognition [DMS target period]), with Group as the between-subject factor and ROI (inferior occipital gyrus, posterior inferior temporal gyrus, fusiform gyrus, anterior inferior temporal gyrus, PRC, hippocampus) as the within-subject factor. Throughout the ROI results (cue and target), we applied the Greenhouse-Geisser correction (Greenhouse \& Geisser, 1959) for nonsphericity of the within-subject variable where necessary, which is indicated by adjusted degrees of freedom.

\section{RESULTS}

\section{Behavioral Results}

\section{Associative Learning}

The number of runs to acquire the pair-associates (averaged across similar and dissimilar pair-associates) was lowest for synesthetes $(M=2.59, S E=0.34)$, followed by young adults $(M=3.68, S E=0.59)$ and older adults $(M=5.47, S E=0.69)$. A one-way ANOVA revealed a significant main effect of Group, $F(2,54)=5.518, p=.007$. 
Table 1. Mean and Standard Error of the Percent Accuracy (Hits and Correct Rejections) in the DPA and DMS Tasks $(n=19$ in Each Group)

\begin{tabular}{lccr}
\hline Hit Rate (Task) & Young Adults, Mean (SE) & Older Adults, Mean (SE) & Synesthetes, Mean (SE) \\
\hline Accuracy (DPA) & $93.09(1.59)$ & $85.69(2.69)$ & $90.87(2.57)$ \\
Accuracy (DMS) & $96.22(1.21)$ & $96.38(1.25)$ & $93.87(1.37)$ \\
\hline
\end{tabular}

Tukey post hoc comparisons showed that synesthetes required significantly fewer runs than older adults $(p=$ $.006 ; d=0.38$; power $=1.11)$, whereas no significant difference was found between young and older adults ( $p=$ $.072 ; d=0.65$; power $=0.56)$ and between young adults and synesthetes $(p=.585 ; d=0.38$; power $=0.78)$.

\section{DPA and DMS during Scanning}

Accuracy was high in both tasks and comparable across groups (see Table 1). A $3 \times 2$ between-subject ANOVA with Group and Task as factors yielded no significant main effect of Group, $F(2,108)=2.048, p=.134$, $\eta_{\mathrm{p}}^{2}=0.037$. A highly significant main effect of Task, $F(1,108)=$ $18.074, p<.001, \eta_{\mathrm{p}}^{2}=0.146$, suggested that the DPA task was more demanding than the DMS task. No significant interaction was found between Group and Task, $F(2,108)=$ 2.059, $p=.133, \eta_{\mathrm{p}}^{2}=0.037$.

\section{fMRI Results}

\section{Associative Retrieval: Cue Period}

ROI results, cue. Figure 3 illustrates the average percent signal change from posterior to anterior ROIs along the ventral visual stream. The ANOVA yielded a significant main effect of Group, $F(2,54)=3.863, p=.027, \eta_{\mathrm{p}}^{2}=$ 0.125 , a significant main effect of ROI, $F(3.80,205,176)=$ $13.24, p<.001, \eta_{\mathrm{p}}^{2}=0.197$, but no significant interaction between Group and ROI, $F(10,270)=1.44, p=$ $.161, \eta_{\mathrm{p}}^{2}=0.051$. We computed pairwise comparisons on the group effect using a Bonferroni correction. Significantly lower activity (averaged across ROIs) was found in synesthetes relative to older adults that was statistically significant $(p=.041)$ and in synesthetes relative to young adults that approached significance $(p=.088)$. No activation difference was found between young and older adults $(p=.948)$. Figure 3 further illustrates that synesthetes had consistently lower mean percent signal change relative to young and/or older adults in posterior ROIs (from the inferior occipital to the anterior inferior temporal gyrus) whereas activity was comparable between groups in anterior regions including the PRC and the hippocampus. In line with our hypothesis, this suggests differential retrieval strategies in synesthetes' posterior visual areas and similar retrieval strategies to the other two groups in anterior-medial brain structures. To examine whether group differences could also be found for the working memory control task, we further analyzed the DMSC images. Figure 4 illustrates the average percent signal change in all ROIs from posterior to anterior regions along the ventral visual stream. The signal in the anterior inferior temporal cortex did not survive the threshold of $p<.005$ (uncorrected) and was therefore extracted from active voxels at a more lenient theshold of $p<.05$ (uncorrected). Results of the $3 \times 6$ mixed ANOVA yielded a significant main effect of ROI, $F(3.76,202.95)=$ $16.01, p<.001, \eta_{\mathrm{p}}^{2}=0.229$, but no main effect of Group, $F(2,54)=1.964, p=.150, \eta_{\mathrm{p}}^{2}=0.068$, and no interaction between Group and ROI, $F(10,270)=1.301, p=$ $.230, \eta_{\mathrm{p}}^{2}=0.046$, indicating that the group effects in the DPA task were specific to associative retrieval. We also computed the difference scores for DPA $>$ DMS for each group and ROI and subjected these to a 3 (Group) $\times 6$ (ROI) mixed ANOVA. The significant main effect of Group disappeared, $F(2,54)=0.459, p=.634$, and there was no significant main effect of ROI, $F(2.44,131.50)=$ $1.255, p=.291$ and no Group $\times$ ROI interaction, $F(10$,
Figure 3. Percent signal change, showing six ROIs plotted for young adults, older adults, and synesthetes during associative retrieval (cue period), averaged across condition (similar, dissimilar). Error bars indicate the SEM. Inf Occ Gyrus = inferior occipital gyrus; Post Inf Temporal $=$ posterior inferior temporal gyrus; Fusiform = fusiform gyrus; Ant Inf Temp = anterior inferior temporal gyrus; $\mathrm{PRC}=$ perirhinal cortex.

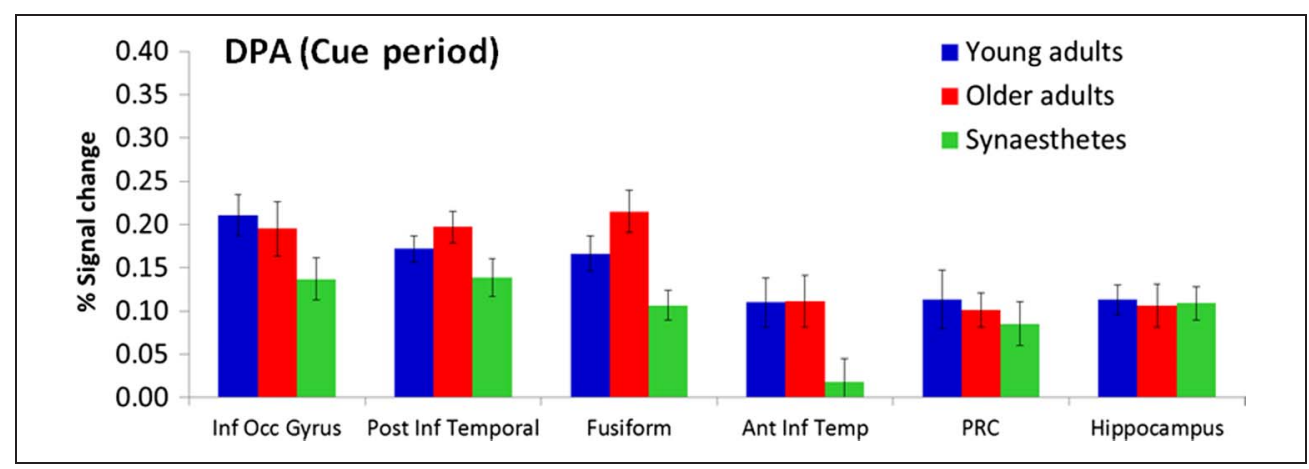


Figure 4. Percent signal change in six ROIs plotted for young adults, older adults, and synesthetes during the cue period of the DMS task. For the anterior inferior temporal gyrus denoted with $a+$ sign, we extracted the percent signal change from active voxels at a more lenient threshold of $p<.05$ (unc.). Error bars indicate the SEM. Inf Occ Gyrus = inferior occipital gyrus; Post Inf Temporal $=$ posterior inferior temporal gyrus; Fusiform $=$ fusiform gyrus; Ant Inf Temp = anterior inferior temporal gyrus; $\mathrm{PRC}=$ perirhinal cortex .

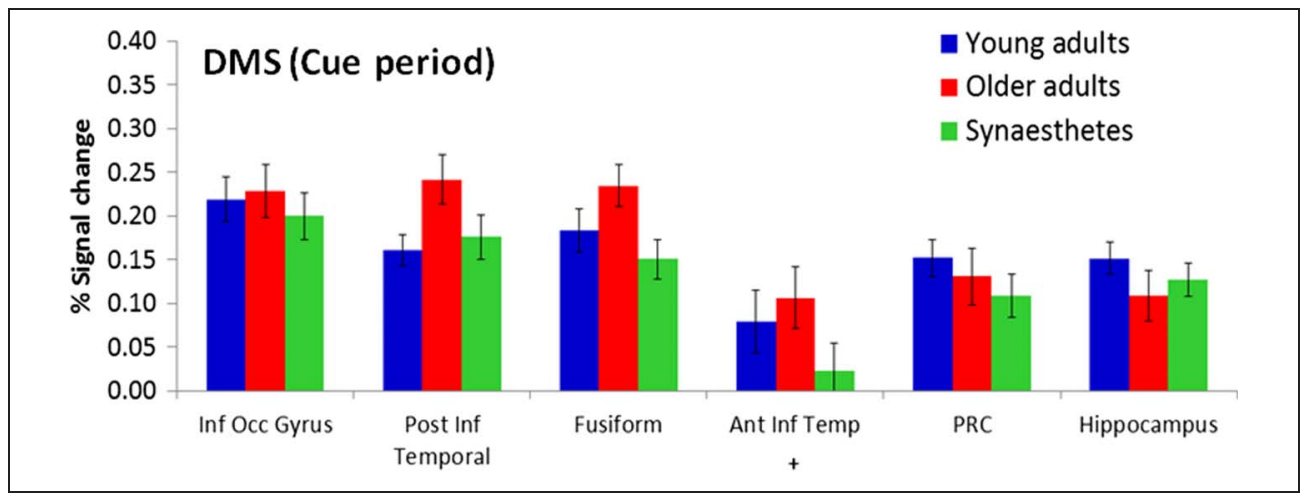

$270)=0.605, p=.810$. The problem with this contrast likely is that both tasks (DPA and DMS) strongly targeted the VVS, leaving activation differences between tasks minimal. This was particularly evident by the nonsignificant main effect of ROI, revealing comparable activation levels across ROIs when DMS activity was subtracted from DPA activity. Hence, group differences in associative retrieval were best illustrated when keeping the two tasks (DPA and DMS) separate.

Whole-brain results, cue. Next, we computed group effects, using the contrast images $\mathrm{sC}>$ DMSC and $\mathrm{dC}>$ DMSC to investigate group differences during associative retrieval while accounting for working memory effects. The one-way ANOVA showed a significant main effect of group in the dissimilar condition within the left calcarine gyrus. No group difference was found in the similar condition. Tukey post hoc tests on the percent signal change of the peak voxel (MNI: $-6-64-19$ ) revealed that the group effect in the dissimilar condition was driven by young adults and synesthetes, who both showed less percent signal change relative to older adults. Post hoc results approached significance for the difference between synesthetes and older adults $(p=.069)$ and young and older adults $(p=.065)$, whereas no difference was found between synesthetes and young adults $(p=.999)$. To evaluate the hypothesis that synesthetes show the most differentiated and older adults the least differentiated activation pattern across groups, we performed post hoc tests for the dissimilar retrieval condition in SPM, using
Figure 5. Post hoc results of associative retrieval. Regions exhibiting enhanced activation in older versus young adults (left) and older adults versus synesthetes (right) during retrieval of dissimilar pairassociates at the cue period. Group contrasts were masked with the task effect $\mathrm{dC}>$ DMSC and suprathresholded at $p<.001$ (uncorrected), $k=5$ voxels.

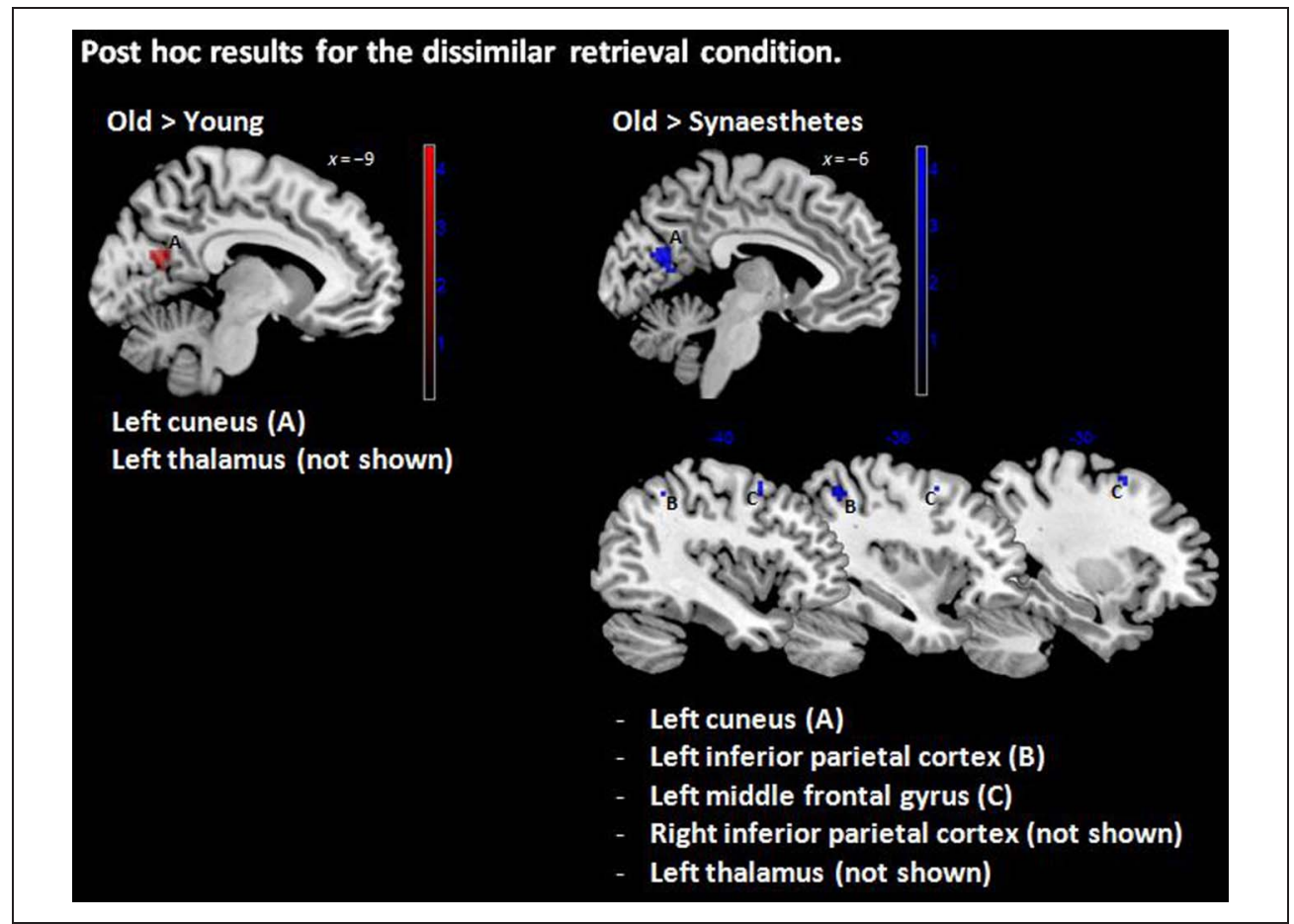


Figure 6. Percent signal change in six ROIs plotted for young adults, older adults, and synesthetes during associative recognition (target period) The percent signal change for each ROI was averaged across condition (similar, dissimilar) and target type (match, nonmatch). Error bars indicate the SEM. Inf Occ Gyrus = inferior occipital gyrus; inferior temporal gyrus; Fusiform = fusiform gyrus; Ant Inf Temp = anterior inferior temporal gyrus; Post Inf Temporal $=$ posterior $\mathrm{PRC}=$ perirhinal cortex

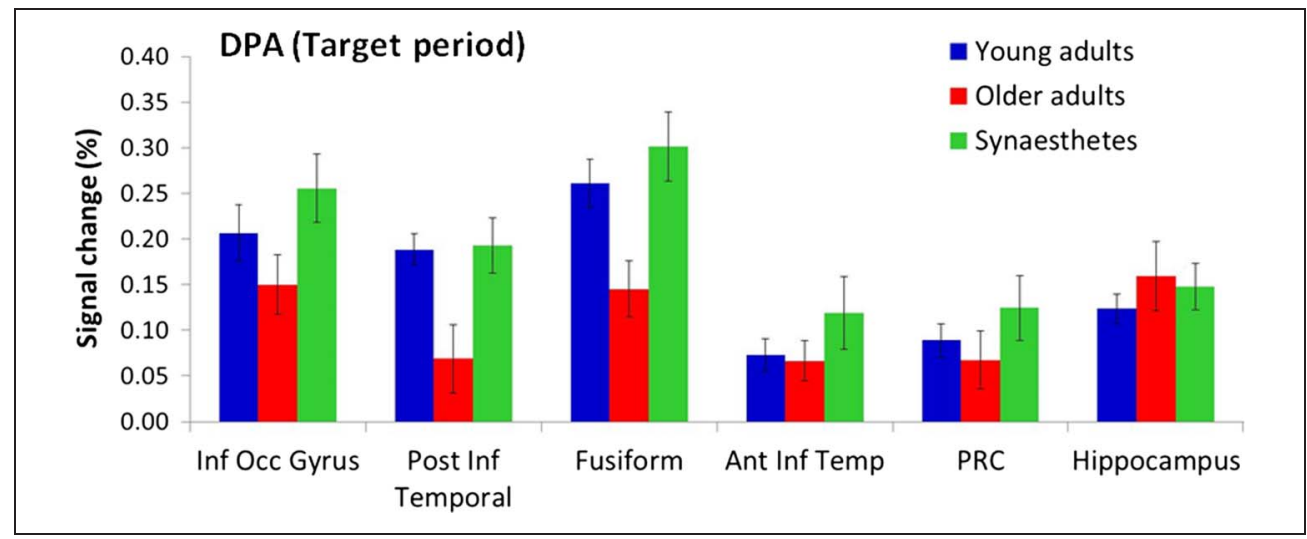

the contrasts old $>$ young, old $>$ synesthetes, young $>$ synesthetes, and the reverse contrasts, respectively. All group comparisons were calculated by inclusively masking the $\mathrm{dC}$ images with task activity $\mathrm{dC}>$ DMSC (see Secondlevel analyses). We found a significant effect for the two contrasts old $>$ young and old $>$ synesthetes (Figure 5 ). Relative to young adults, older adults showed significantly greater activity in the cuneus and thalamus. Relative to synesthetes, however, older adults revealed a more distributed activation pattern, spanning the cuneus, cerebellum, thalamus, bilateral inferior parietal regions, as well as the left middle frontal and precentral gyrus. Thus, although there was no activation difference between young adults and synesthetes, our data reveal that synesthetes showed the most differentiated and efficient retrieval system, which could only be detected against older adults. No group difference was found for the similar condition, suggesting comparable retrieval strategies across groups in the low-memory load condition.

\section{Associative Recognition: Target Period}

ROI results, target. Figure 6 illustrates that synesthetes showed greater mean percent signal change relative to young and older adults in all regions apart from the hippocampus, demonstrating enhanced sensitivity relative to
Figure 7. Main effect of group during associative recognition (target period) shown in the cuneus (BA 17; top) and the left lingual gyrus (BA 17; bottom). Left (top and bottom): The main effects of group rendered on the individual subjects' brain available in MRIcron. Right: The percent signal change averaged across activity in the cuneus (top) and the left lingual gyrus (bottom) for young adults, older adults, and synesthetes, in response to Sim match, Sim nonmatch, Diss match, and Diss nonmatch trials. Error bars indicate the SEM. Asterisks indicate significant group differences derived from Tukey post hoc tests conducted for separate oneway ANOVAs for Sim match, Sim nonmatch, Diss match, Diss nonmatch. Sim = similar; Diss $=$ dissimilar

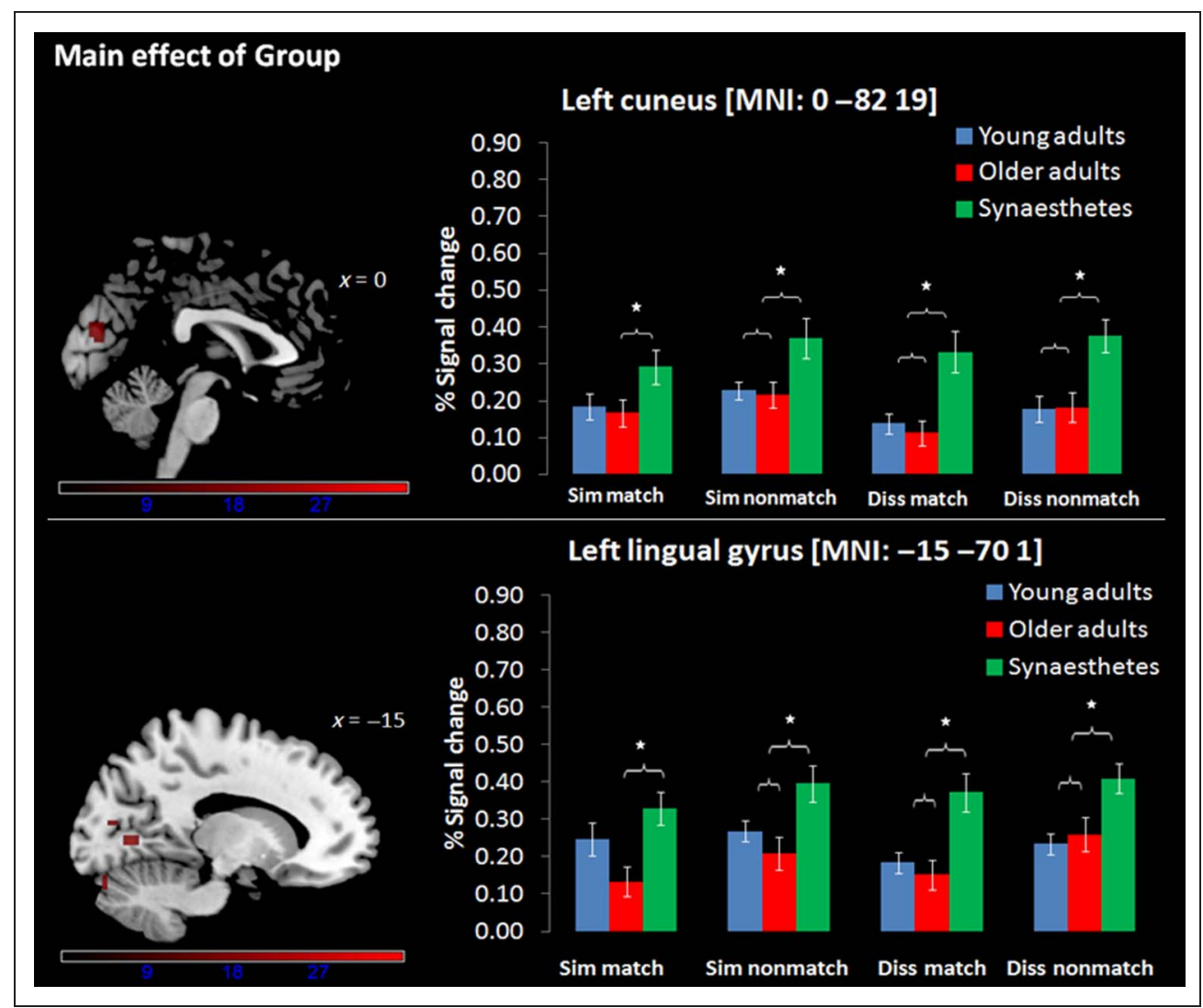


Figure 8. Percent signal change in six ROIs plotted for young adults, older adults, and synesthetes during the working memory task (DMS, target period). The percent signal change for each ROI was averaged across target type (match, nonmatch). Error bars indicate the SEM. Inf Occ Gyrus $=$ inferior occipital gyrus; Post Inf Temporal $=$ posterior inferior temporal gyrus; Fusiform = fusiform gyrus; Ant Inf Temp = anterior inferior temporal gyrus; $\mathrm{PRC}=$ perirhinal cortex

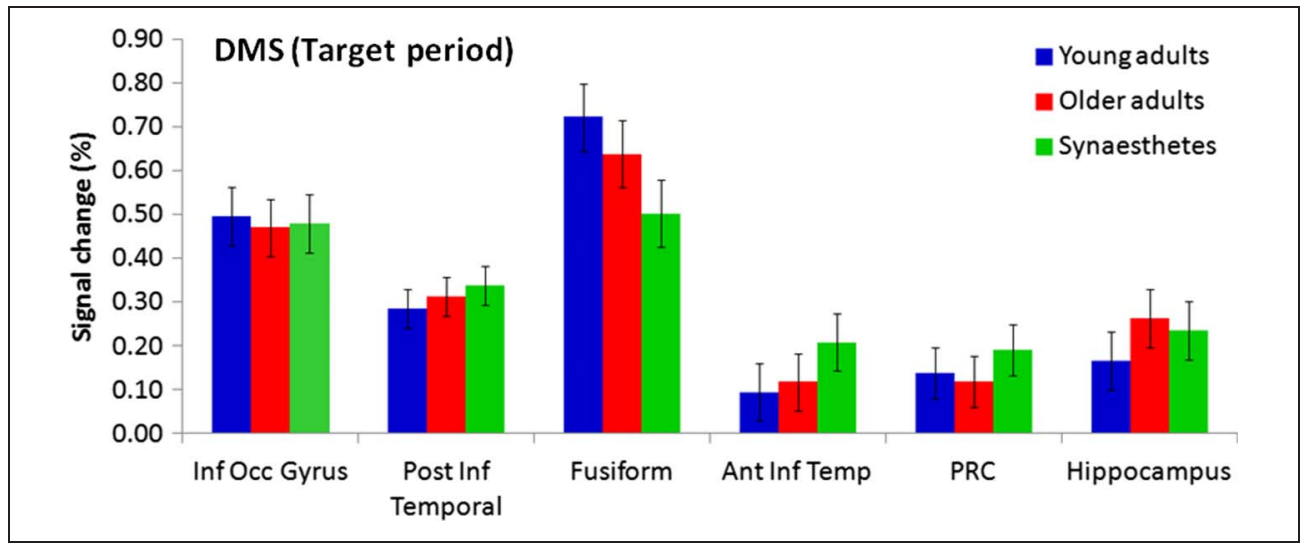

the other two groups in response to target recognition. The 3 (Group) $\times 6$ (ROI) mixed ANOVA yielded a significant main effect of Group $F(2,54)=4.545, p=$ $.015, \eta_{\mathrm{p}}^{2}=0.144$, and a significant Group $\times$ ROI interaction, $F(10,270)=1.962, p=.038, \eta_{p}^{2}=0.068$. To demonstrate the group differences, pairwise group comparisons were computed using the Bonferroni correction. Synesthetes showed significantly greater signal change relative to older adults $(p=.012)$, whereas no significant difference was found between synesthetes and young adults $(p=.665)$ and between young and older adults $(p=.251)$. Next, we analyzed the DMST images of the working memory control task. Figure 8 illustrates the average percent signal change in all ROIs from posterior to anterior regions for the DMS task. Results of the $3 \times 6$ mixed ANOVA yielded a significant main effect of ROI, $F(5,270)=31.95, p<.001, \eta_{\mathrm{p}}^{2}=$ 0.372 , but no main effect of Group, $F(2,54)=0.015$, $p=.958, \eta_{\mathrm{p}}^{2}=0.001$, and no interaction between Group and ROI, $F(10,270)=1.174, p=.309, \eta_{\mathrm{p}}^{2}=0.042$, indicating that the group effects of the DPA task were spe- cific to associative recognition (Figure 7). We further subjected the difference scores of the DPA and DMS tasks (DPA > DMS) to a 3 (group) $\times 6$ (ROI) mixed ANOVA. Results yielded no significant main effect of Group, $F(2$, $54)=0.204, p=.816, \eta_{\mathrm{p}}^{2}=0.008$, similar to the DPA $>$ DMS results of the cue period. However, a significant main effect of ROI, $F(5,270)=9.302 ; p<.001, \eta_{\mathrm{p}}^{2}=0.147$, demonstrated that the DMS target images generally yielded greater activation than the DPA target images and yielded no Group $\times$ ROI interaction, $F(10,270)=0.770$, $p=.657, \eta_{\mathrm{p}}^{2}=0.028$ (cf. Figures 7 and 8).

Whole-brain results, target. Next, we computed group comparisons for the target period to investigate group differences in associative recognition. $F$ tests yielded a significant main effect of Group on associative recognition in posterior visual regions (Table 2) but no significant interaction between group and condition; between group and target type; or between group, condition, and target type. To examine the group differences more closely,

Table 2. Brain Regions with a Significant Main Effect of Group at Associative Recognition

\begin{tabular}{|c|c|c|c|c|c|}
\hline \multirow[b]{2}{*}{ Brain Region } & \multicolumn{3}{|c|}{ MNI Coordinates } & \multirow[b]{2}{*}{$F$} & \multirow{2}{*}{$\begin{array}{c}\text { Cluster Size } \\
\text { (Voxels) }\end{array}$} \\
\hline & $x$ & $y$ & $z$ & & \\
\hline Left cuneus & 0 & -82 & 19 & 20.68 & 83 \\
\hline Left calcarine gyrus & -12 & -85 & 13 & 19.28 & \\
\hline Right calcarine gyrus & 18 & -76 & 4 & 17.50 & \\
\hline Left cerebellum & -42 & -73 & -23 & 35.69 & 42 \\
\hline Left lingual gyrus & -15 & -70 & 1 & 20.79 & 17 \\
\hline Left calcarine gyrus & -24 & -61 & 4 & 14.80 & \\
\hline Left cerebellum & -15 & -85 & -23 & 20.29 & 12 \\
\hline Right lingual gyrus & 39 & -79 & -17 & 17.76 & 8 \\
\hline Right inferior occipital gyrus & 36 & -82 & -11 & 14.83 & \\
\hline
\end{tabular}

MNI coordinates represent the location of the peak voxels. The peak voxels of each cluster with the cluster size are followed by separate maxima ( $8 \mathrm{~mm}$ apart) within the cluster. Results were thresholded at $p<.05$, FWE-corrected with a minimum cluster size of 5 voxels. 
Table 3. Post hoc Results for Associative Recognition (Target Period) of Dissimilar Pairs Contrasted against DMS (dT > DMST)

\begin{tabular}{|c|c|c|c|c|c|}
\hline \multirow{2}{*}{$\frac{\text { Group Contrast }}{\text { Brain Region }}$} & \multicolumn{3}{|c|}{ MNI Coordinates } & \multirow[b]{2}{*}{$t$} & \multirow{2}{*}{$\begin{array}{c}\text { Cluster Size } \\
\text { (Voxels) }\end{array}$} \\
\hline & $x$ & $y$ & $z$ & & \\
\hline \multicolumn{6}{|l|}{ Old $>$ Young } \\
\hline Right hippocampus & 36 & -13 & -14 & 4.99 & 29 \\
\hline Left inferior frontal gyrus & -45 & 23 & 16 & 3.8 & 16 \\
\hline Right middle frontal gyrus & 33 & 14 & 19 & 4.58 & 14 \\
\hline Left middle frontal gyrus & -33 & 44 & 16 & 3.96 & 10 \\
\hline Left hippocampus & -27 & -7 & -14 & 3.68 & 10 \\
\hline Right inferior frontal gyrus & 54 & 17 & 22 & 3.51 & 5 \\
\hline Right anterior cingulate cortex & 12 & 47 & 16 & 3.83 & 5 \\
\hline Left inferior temporal gyrus & -57 & -58 & -11 & 3.36 & 5 \\
\hline \multicolumn{6}{|l|}{ Old $>$ Synesthetes } \\
\hline Left inferior frontal gyrus & -42 & 26 & 16 & 3.82 & 16 \\
\hline Right inferior frontal gyrus & 39 & 35 & -8 & 4.15 & 6 \\
\hline \multicolumn{6}{|l|}{ Synesthetes > Young } \\
\hline Left thalamus & -9 & -16 & 7 & 3.99 & 9 \\
\hline Left hippocampus & -18 & -37 & 1 & 4.01 & 9 \\
\hline Right fusiform gyrus & 24 & -46 & -2 & 3.75 & 6 \\
\hline Left fusiform gyrus & -24 & -79 & 4 & 3.56 & 5 \\
\hline
\end{tabular}

MNI coordinates represent the location of the peak voxels. The peak voxels of each cluster with the cluster size are followed by separate maxima ( $8 \mathrm{~mm}$ apart) within the cluster. Results were thresholded at $p<.001$ (uncorrected) with a minimum cluster size of 5 voxels.

we computed Tukey post hoc tests for three relevant cluster maxima in occipitotemporal cortex (see Table 2): the left cuneus and the left and right lingual gyrus. To this end, we extracted the average percent signal change for each cluster and group and performed three $3 \times 2 \times 2$ mixed ANOVAs with Group, Condition, and Target type as factors. In line with our prediction, we found greater average percent signal change in each condition and target type for synesthetes than for young and older adults in the left cuneus and the left lingual gyrus (Figure 7). Tukey post hoc tests for the average signal across Condition and Target type showed a significant difference between synesthetes and young adults (both clusters, $p<.01$ ), synesthetes and older adults (both clusters, $p<.01$ ), but not between young and older adults (both clusters, $p>.05$ ). In the right lingual gyrus, the average percent signal change was higher in synesthetes $(M=0.65 \%, S E=0.08)$ and young adults $(M=0.59 \%$, $S E=0.08)$ relative to older adults $(M=0.41 \%, S E=$ 0.08). However, the post hoc test yielded no significant difference between groups $(p>.05)$, although the difference between synesthetes and older adults approached significance $(p=.086)$. Next, we examined group differences during associative recognition while contrasting against the
DMS task to account for perceptual and working memory effects (contrast images dT $>$ DMST and s'T $>$ DMST). A significant main effect of Group was found for the dissimilar condition in the right hippocampus and right middle frontal gyrus. Post hoc tests revealed an effect of synesthesia, showing a significant difference for the contrast synesthetes $>$ young adults, with synesthetes showing enhanced activation in the left hippocampus, bilateral fusiform gyrus, and left thalamus (Table 3). We also found significant age differences for the contrasts old $>$ young and old $>$ synesthetes (Table 3 ). The similar condition yielded a significant group effect in regions of the left auditory cortex (Heschl's gyrus, superior and middle temporal gyrus), bilateral putamen, and left postcentral gyrus. Post hoc tests for the similar condition revealed an effect of age, showing that older adults activated these regions significantly more than young adults and synesthetes, whereas all other pairwise comparisons were nonsignificant.

\section{DISCUSSION}

Our study investigated whether differences in sensoryperceptual abilities, as observed in individuals with 
synesthesia and older adults, translated into visual associative memory for non-synesthesia-inducing stimuli. We observed evidence of enhanced sensitivity in synesthetes, which was manifest as (i) a behavioral associative learning advantage, (ii) a more differentiated activation pattern during associative retrieval, and (iii) enhanced activity in hippocampus, fusiform, and visual cortex during associative recognition. Previous EEG studies have shown evidence of enhanced processing in the primary visual cortex in synesthetes, indicative of increased sensitivity to visual stimuli (Barnett et al., 2008). The fMRI data acquired in this study, which demonstrate an effect of synesthesia on activation in early visual regions, also suggest that sensory processing of non-synesthesia-inducing stimuli differs in synesthetes for both perceptual and memory tasks. These findings can be considered in light of the representational account of memory (Bussey \& Saksida, 2007), which envisages the neural substrates of visual perception and memory to be on a continuum. According to this account, enhanced sensitivity to our fractal images at the point of perception would be predicted to translate into a learning and memory advantage. Indeed, the effects of synesthesia were most evident when bottomup perceptual cues were available (i.e., at associative learning and recognition). For instance, in the associative learning paradigm, stimuli were presented a priori and perceptually reinstated during the forced-choice task. Enhanced sensory processing might have translated into a learning advantage in synesthetes who required significantly fewer runs than older adults to reach criterion. This effect was not seen in young relative to older adults, indicating that synesthetes were most efficient in extracting and memorising bottom-up perceptual cues. Support for this claim comes from neurophysiological studies, showing that visual long-term memory is associated with long-term potentiation (LTP), which can be induced in primary visual cortex (Artola \& Singer, 1987; Komatsu, Toyama, Maeda, \& Sakaguchi, 1981). Critical to the induction of LTP is the reduced GABAergic inhibition in visual cortex (Artola \& Singer, 1987). Interestingly, synesthetes were found to show hyperexcitability in primary visual cortex (Terhune et al., 2011), perhaps due to a facilitation of LTP induction following visual stimulation (but see Terhune, Song, Duta, \& Kadosh, 2014). Using magnetic resonance spectroscopy and TMS, another study (Terhune et al., 2015) found a relationship between glutamate, but not GABA levels, and phosphene perception in early visual cortex: Across synesthetes and controls, higher glutamate levels were associated with lower thresholds of phosphene perception following TMS application. Of the two groups, however, the synesthetes' phosphene threshold was significantly lower than that of controls. Thus, neurochemical concentrations in synesthetes' visual cortex could explain our synesthetes' enhanced associative learning, where bottom-up perceptual cues were available. The fact that we found no behavioral group difference at associative retrieval can be attributed to the high criterion to which the pair-associates had to be learned (87.5\%). Specifically, our associative learning paradigm was selfpaced, therefore meeting the different encoding demands required with age and synesthesia for later successful retrieval. The fMRI results of our recognition phase extend Terhune et al.'s (2011) finding of enhanced cortical excitability in synesthetes from the perceptual to the memory domain. We found a group effect in early visual regions, with synesthetes showing significantly greater signal changes relative to the other two groups in the cuneus and left lingual gyrus. Our ROI results further revealed significantly greater signal change in synesthetes relative to nonsynesthetes in posterior regions of the VVS. Notably, as in the associative learning task, our recognition task directed participants toward the visual properties of the targets to make a match or nonmatch decision. Thus, the synesthetes' greater visual cortex activity in response to these stimuli suggests enhanced excitability during target recognition. Moreover, we found enhanced activation in synesthetes relative to young adults in the fusiform gyrus and hippocampus when working memory processes were subtracted from associative recognition. This suggests that, even after accounting for the synesthetes' enhanced sensory processing of bottom-up perceptual targets, they continued to show higher activation in anterior, memoryrelated regions that are directly relevant to associative recognition (i.e., recalling the cue and matching or rejecting the target). Our finding of the synesthetes' enhanced activation at recognition fits the computations of the visual system more comfortably than our alternative hypothesis, which stated that synesthetes might show reduced activation at recognition. Reduced activation often reflects a well-differentiated brain area, in which few but dedicated units respond to specific classes of stimuli (Kok et al., 2012). Given the BOLD in fMRI, smaller firing units would therefore induce less signal change and emerge as a "less active" area. However, because we did not use images that are known to stimulate well-differentiated areas (e.g., faces taxing the fusiform gyrus, or, specific to synesthetes, letters activating color area V4; Brang, Hubbard, Coulson, Huang, \& Ramachandran, 2010; Hubbard, Arman, Ramachandran, \& Boynton, 2005; Nunn et al., 2002), reduced activation was less likely to be found in our experiment. As demonstrated by Martins et al. (2014), fractals are represented along the VVS rather than by a single brain region. Hence, the synesthetes' enhanced activation in early visual regions, presumably the result of hyperexcitability, suggests superior sensory processing of bottom-up perceptual targets.

A different pattern emerged from the group comparisons found at associative retrieval (cue presentation). Although we found a main effect of group in early visual cortex (as during recognition), the pattern of activation was reversed: Synesthetes, as well as young adults, showed lower calcarine gyrus activity relative to older adults at retrieval and higher activity during recognition. This was supported by the ROI results, showing reduced 
activity in occipital-temporal areas during retrieval and enhanced activity during recognition in synesthetes versus older adults. We interpret this result as evidence for reduced sensitivity in older adults' primary visual cortex, in line with other studies (Peiffer et al., 2009; Justino, Kergoat, \& Kergoat, 2001; Levine et al., 2000; Ross et al., 1997). Although these studies typically found reduced visual cortex activity in older adults using bottom-up perceptual detection tasks, the enhanced activity found in our group of older adults during associative retrieval suggests that top-down memory processes require enhanced activity to compensate degraded sensory functions. In other words, the reversed group effect observed between associative retrieval and recognition indicates that reduced sensitivity in visual cortex (as in aging) comes with an activation decrease during bottom-up perceptual processing and an activation increase during top-down retrieval, whereas enhanced sensitivity (as in synesthesia) shows the opposite pattern. The results of our post hoc tests also revealed greater activity in older adults relative to synesthetes in parietal and frontal regions, over and above the enhanced activity in the cuneus, an effect that was not found for the comparison of older versus young adults. This demonstrates a subtle effect of synesthesia and provides evidence for a more differentiated retrieval system that can only be detected against older adults. The fact that no differences were found between young adults and synesthetes could be attributed to our non-synesthesiainducing stimuli; the black-and-white fractal images were expected to trigger group effects related to differences in visual sensitivity, which differs most between synesthetes (enhanced sensitivity; Barnett et al., 2008) and older adults (reduced sensitivity; Goh, 2011). These findings support the sensitivity hypothesis and are consistent with the representational account of memory: Enhanced sensitivity in early visual cortex may accentuate stimulus processing along the VVS in synesthetes and reduce top-down control required from frontal and parietal regions. Future connectivity studies should investigate whether differential processing functions in early visual cortex may have a pervasive effect on the entire retrieval network. Moreover, further research should address the limitations of our cross-sectional design, in which correlations between sensory perceptual and cognitive functioning might be exaggerated because of the uniformity within specific age cohorts (La Fleur \& Salthouse, 2014; Lindenberger \& Ghisletta, 2009; Valentijn et al., 2005; Hofer, Berg, \& Era, 2003). Future longitudinal designs with synesthetes and nonsynesthetes could clarify the developmental trajectories in ventral visual regions as they relate to perception and memory.

In conclusion, our results revealed activation patterns of visual associative memory (retrieval and recognition) that reflect differences in visual perception and memory between synesthetes, young adults, and older adults. To our knowledge, this is the first fMRI study to investigate the neural correlates of memory in synesthetes, allowing us to examine the influence of perception on memory. Our data suggest that the synesthetes' memory advantage for non-synesthesia-inducing stimuli was driven by enhanced visual sensitivity. Behaviorally, this was demonstrated by faster learning of pair-associates relative to older adults. In fMRI, group differences relating to associative retrieval and recognition were mainly found in early visual regions. Specifically, the synesthetes' enhanced sensitivity in visual cortex gave rise to a more differentiated neural system during retrieval, when processing was directed to internal representations of associations. By contrast, during recognition, synesthetes showed enhanced activity in early visual regions, reflecting enhanced sensitivity to external, behaviorally relevant stimuli. Our data further show that synesthesia can inform age differences in perception and memory, with potential translational and clinical implications. Synesthetes differed most strongly from older adults in posterior, sensory regions of the VVS. This converges with poorer age-related, and enhanced synesthesia-related, sensory processing in visual cortex (McDonough et al., 2014; Park et al., 2010). The novelty of our data is that sensory-perceptual processing differences inherent to the three groups translated into differences in visual memory processing, given that participants performed a memory test. This perceptualmnemonic continuum, here demonstrated on the basis of synesthesia and aging, is in line with the representational account (Bussey \& Saksida, 2007).

\section{Acknowledgments}

This work was funded by an MRC PhD studentship, the Brighton and Sussex Medical School, and the Brighton and Sussex University Hospital Trust. J. W. was supported by the Economic and Social Research Council. We thank L. Minati and F. Scheler for discussions and help with programming.

Reprint requests should be sent to Gaby Pfeifer, Brighton and Sussex Medical School, Clinical Imaging Sciences Centre, University of Sussex, Brighton BN1 9RR, United Kingdom, or via e-mail: g.pfeifer@bsms.ac.uk.

\section{REFERENCES}

Artola, A., \& Singer, W. (1987). Long-term potentiation and NMDA receptors in rat visual-cortex. Nature, 330, 649-652.

Barnett, K. J., Foxe, J. J., Molholm, S., Kelly, S. P., Shalgi, S., Mitchell, K. J., et al. (2008). Differences in early sensoryperceptual processing in synesthesia: A visual evoked potential study. Neuroimage, 43, 605-613.

Brang, D., Hubbard, E. M., Coulson, S., Huang, M., \& Ramachandran, V. S. (2010). Magnetoencephalography reveals early activation of $\mathrm{V} 4$ in grapheme-color synesthesia. Neuroimage, 53, 268-274.

Buchsbaum, B. R., Lemire-Rodger, S., Fang, C., \& Abdi, H. (2012). The neural basis of vivid memory is patterned on perception. Journal of Cognitive Neuroscience, 24, 1867-1883.

Bussey, T. J., \& Saksida, L. M. (2007). Memory, perception, and the ventral visual-perirhinal-hippocampal stream: Thinking outside of the boxes. Hippocampus, 17, 898-908.

Daselaar, S. M., Porat, Y., Huijbers, W., \& Pennartz, C. M. A. (2010). Modality-specific and modality-independent 
components of the human imagery system. Neuroimage, 52, 677-685.

Davis, S. W., Dennis, N. A., Daselaar, S. M., Fleck, M. S., \& Cabeza, R. (2008). Que PASA? The posterior-anterior shift in aging. Cerebral Cortex, 18, 1201-1209.

Eagleman, D. M., Kagan, A. D., Nelson, S. S., Sagaram, D., \& Sarma, A. K. (2007). A standardized test battery for the study of synesthesia. Journal of Neuroscience Methods, 159, 139-145.

Eichenbaum, H. (2013). What H.M. taught us. Journal of Cognitive Neuroscience, 25, 14-21.

Eickhoff, S. B., Stephan, K. E., Mohlberg, H., Grefkes, C., Fink, G. R., Amunts, K., et al. (2005). A new SPM toolbox for combining probabilistic cytoarchitectonic maps and functional imaging data. Neuroimage, 25, 1325-1335.

Folstein, M. F., Folstein, S. E., \& McHugh, P. R. (1975). Mini-mental state: A practical method for grading the cognitive state of patients for the clinician. Journal of Psychiatric Research, 12, 189-198.

Friston, K. J., Fletcher, P., Josephs, O., Holmes, A., Rugg, M. D., \& Turner, R. (1998). Event-related fMRI: Characterizing differential responses. Neuroimage, 7, 30-40.

Gläscher, J. (2009). Visualization of group inference data in functional neuroimaging. Neuroinformatics, 7, 73-82.

Goh, J. O. S. (2011). Functional dedifferentiation and altered connectivity in older adults: Neural accounts of cognitive aging. Aging and Disease, 2, 30-48.

Greenhouse, S. W., \& Geisser, S. (1959). On methods in the analysis of profile data. Psychometrika, 24, 95-112.

Gross, V. C., Neargarder, S., Caldwell-Harris, C. L., \& CroninGolomb, A. (2011). Superior encoding enhances recall in color-graphemic synesthesia. Perception, 40, 196-208.

Hofer, S. M., Berg, S., \& Era, P. (2003). Evaluating the interdependence of aging-related changes in visual and auditory acuity, balance, and cognitive functioning. Psychology and Aging, 18, 285-305.

Holdstock, J. S., Hocking, J., Notley, P., Devlin, J. T., \& Price, C. J. (2009). Integrating visual and tactile information in the perirhinal cortex. Cerebral Cortex, 19, 2993-3000.

Hubbard, E. M., Arman, A. C., Ramachandran, V. S., \& Boynton, G. M. (2005). Individual differences among grapheme-color synesthetes: Brain-behavior correlations. Neuron, 45, 975-985.

Hubbard, E. M., Brang, D., \& Ramachandran, V. S. (2011). The cross-activation theory at 10. Journal of Neuropsychology, 5, $152-177$.

Humes, L. E., Busey, T. A., Craig, J., \& Kewley-Port, D. (2013). Are age-related changes in cognitive function driven by age-related changes in sensory processing? Attention, Perception, \& Psychophysics, 75, 508-524.

Justino, L., Kergoat, H., \& Kergoat, M. J. (2001). Changes in the retinocortical evoked potentials in subjects 75 years of age and older. Clinical Neurophysiology, 112, 1343-1348.

Kalkstein, J., Checksfield, K., Bollinger, J., \& Gazzaley, A. (2011). Diminished top-down control underlies a visual imagery deficit in normal aging. Journal of Neuroscience, 31, 15768-15774.

Kok, P., Jehee, J. F. M., \& de Lange, F. P. (2012). Less is more: Expectation sharpens representations in the primary visual cortex. Neuron, 75, 265-270.

Komatsu, Y., Toyama, K., Maeda, J., \& Sakaguchi, H. (1981). Long-term potentiation investigated in a slice preparation of striate cortex of young kittens. Neuroscience Letters, 26, 269-274.

La Fleur, C. G., \& Salthouse, T. A. (2014). Out of sight, out of mind? Relations between visual acuity and cognition. Psychonomic Bulletin E Review, 21, 1202-1208.

Lemaitre, H., Crivello, F., Grassiot, B., Alperovitch, A., Tzourio, C., \& Mazoyer, B. (2005). Age- and sex-related effects on the neuroanatomy of healthy elderly. Neuroimage, 26, 900-911.
Levine, B. K., Beason-Held, L. L., Purpura, K. P., Aronchick, D. M., Optican, L. M., Alexander, G. E., et al. (2000). Age-related differences in visual perception: A PET study. Neurobiology of Aging, 21, 577-584.

Likert, R. (1932). A technique for the measurement of attitudes. Archives of Psychology, 140, 1-55.

Lindenberger, U., \& Ghisletta, P. (2009). Cognitive and sensory declines in old age: Gauging the evidence for a common cause. Psychology and Aging, 24, 1-16.

Luders, E., Steinmetz, H., \& Jancke, L. (2002). Brain size and grey matter volume in the healthy human brain. NeuroReport, 13, 2371-2374.

Maldjian, J. A., Laurienti, P. J., Kraft, R. A., \& Burdette, J. H. (2003). An automated method for neuroanatomic and cytoarchitectonic atlas-based interrogation of fMRI data sets. Neuroimage, 19, 1233-1239.

Martins, M. J., Fischmeister, F. P., Puig-Waldmuller, E., Oh, J., Geissler, A., Robinson, S., et al. (2014). Fractal image perception provides novel insights into hierarchical cognition. Neuroimage, 96, 300-308.

Mayes, A., Montaldi, D., \& Migo, E. (2007). Associative memory and the medial temporal lobes. Trends in Cognitive Sciences, 11, 126-135.

McDonough, I. M., Cervantes, S. N., Gray, S. J., \& Gallo, D. A. (2014). Memory's aging echo: Age-related decline in neural reactivation of perceptual details during recollection. Neuroimage, 98, 346-358.

Nunn, J. A., Gregory, L. J., Brammer, M., Williams, S. C. R., Parslow, D. M., Morgan, M. J., et al. (2002). Functional magnetic resonance imaging of synesthesia: Activation of V4/V8 by spoken words. Nature Neuroscience, 5, 371-375.

Park, D. C., \& McDonough, I. M. (2013). The dynamic aging mind: Revelations from functional neuroimaging research. Perspectives on Psychological Science, 8, 62-67.

Park, D. C., Polk, T. A., Park, R., Minear, M., Savage, A., \& Smith, M. R. (2004). Aging reduces neural specialization in ventral visual cortex. Proceedings of the National Academy of Sciences, U.S.A., 101, 13091-13095.

Park, J., Carp, J., Hebrank, A., Park, D. C., \& Polk, T. A. (2010). Neural specificity predicts fluid processing ability in older adults. Journal of Neuroscience, 30, 9253-9259.

Park, J., Carp, J., Kennedy, K. M., Rodrigue, K. M., Bischof, G. N., Huang, C. M., et al. (2012). Neural broadening or neural attenuation? Investigating age-related dedifferentiation in the face network in a large lifespan sample. Journal of Neuroscience, 32, 2154-2158.

Peiffer, A. M., Hugenschimidt, C. E., Maldjian, J. A., Casanova, R., Srikanth, R., Hayasaka, S., et al. (2009). Aging and the interaction of sensory cortical function and structure. Human Brain Mapping, 30, 228-240.

Pfeifer, G., Rothen, N., Ward, J., Chan, D., \& Sigala, N. (2014). Associative memory advantage in grapheme-color synesthetes compared to older, but not young adults. Frontiers in Psychology, 5, 696.

Pritchard, J., Rothen, N., Coolbear, D., \& Ward, J. (2013). Enhanced associative memory for colour (but not shape or location) in synaesthesia. Cognition, 127, 230-234.

Radvansky, G. A., Gibson, B. S., \& McNerney, M. W. (2011). Synesthesia and memory: Color congruency, von Restorff, and false memory effects. Journal of Experimental Psychology: Learning, Memory, and Cognition, 37, 219-229.

Ramachandran, V. S., \& Hubbard, E. M. (2001). Psychophysical investigations into the neural basis of synaesthesia. Proceedings of the Royal Society B: Biological Sciences, 268, 979-983.

Ranganath, C., Cohen, M. X., Dam, C., \& D'Esposito, M. (2004). Inferior temporal, prefrontal, and hippocampal contributions 
to visual working memory maintenance and associative memory retrieval. Journal of Neuroscience, 24, 3917-3925.

Raz, N., Lindenberger, U., Rodrigue, K. M., Kennedy, K. M., Head, D., Williamson, A., et al. (2005). Regional brain changes in aging healthy adults: General trends, individual differences and modifiers. Cerebral Cortex, 15, 1676-1689.

Ross, M. H., YurgelunTodd, D. A., Renshaw, P. F., Maas, L. C., Mendelson, J. H., Mello, N. K., et al. (1997). Age-related reduction in functional MRI response to photic stimulation. Neurology, 48, 173-176.

Rothen, N., \& Meier, B. (2010). Grapheme-colour synaesthesia yields an ordinary rather than extraordinary memory advantage: Evidence from a group study. Memory, 18, 258-264.

Rothen, N., Meier, B., \& Ward, J. (2012). Enhanced memory ability: Insights from synaesthesia. Neuroscience $\mathcal{E}$ Biobehavioral Reviews, 36, 1952-1963.

Rothen, N., Seth, A. K., Witzel, C., \& Ward, J. (2013). Diagnosing synaesthesia with online colour pickers: Maximising sensitivity and specificity. Journal of Neuroscience Methods, 215, 156-160.

Saksida, L. M., \& Bussey, T. J. (2010). The representationalhierarchical view of amnesia: Translation from animal to human. Neuropsychologia, 48, 2370-2384.

Schott, B. H., Henson, R. N., Richardson-Klavehn, A., Becker, C., Thoma, V., Heinze, H. J., et al. (2005). Redefining implicit and explicit memory: The functional neuroanatomy of priming, remembering, and control of retrieval. Proceedings of the National Academy of Sciences, U.S.A., 102, 1257-1262.

Squire, L. R., \& Wixted, J. T. (2011). The cognitive neuroscience of human memory since HM. Annual Review of Neuroscience, 34, 259-288.

Staresina, B. P., \& Davachi, L. (2010). Object unitization and associative memory formation are supported by distinct brain regions. Journal of Neuroscience, 30, 9890-9897.

St-Laurent, M., Abdi, H., Bondad, A., \& Buchsbaum, B. R. (2014). Memory reactivation in healthy aging: Evidence of stimulus-specific dedifferentiation. Journal of Neuroscience, 34, 4175-4186.
Terhune, D. B., Murray, E., Near, J., Stagg, C. J., Cowey, A., \& Kadosh, R. C. (2015). Phosphene perception relates to visual cortex glutamate levels and covaries with atypical visuospatial awareness. Cerebral Cortex, 25, 4341-4350.

Terhune, D. B., Song, S. M., Duta, M. D., \& Kadosh, R. C. (2014). Probing the neurochemical basis of synaesthesia using psychophysics. Frontiers in Human Neuroscience, 8 , Article 89

Terhune, D. B., Tai, S., Cowey, A., Popescu, T., \& Kadosh, R. C. (2011). Enhanced cortical excitability in grapheme-color synesthesia and its modulation. Current Biology, 21, 2006-2009.

Valentijn, S. A. M., van Boxtel, M. P. J., van Hooren, S. A. H., Bosma, H., Beckers, H. J. M., Ponds, R. W. H. M., et al. (2005). Change in sensory functioning predicts change in cognitive functioning: Results from a 6-year follow-up in the Maastricht aging study. Journal of the American Geriatrics Society, 53, 374-380.

Wang, Z. C., Yao, Z. M., Yuan, N. N., Liang, Z., Li, G. X., \& Zhou, Y. F. (2014). Declined contrast sensitivity of neurons along the visual pathway in aging cats. Frontiers in Aging Neuroscience, 6, Article 163.

Ward, J. (2013). Synesthesia. Annual Review of Psychology, 64, 2.1-2.27.

Ward, J., Hovard, P., Jones, A., \& Rothen, N. (2013). Enhanced recognition memory in grapheme-color synaesthesia for different categories of visual stimuli. Frontiers in Psychology, 4, 762.

Wheeler, M. E., Petersen, S. E., \& Buckner, R. L. (2000). Memory's echo: Vivid remembering reactivates sensoryspecific cortex. Proceedings of the National Academy of Sciences, U.S.A., 97, 11125-11129.

Yaro, C., \& Ward, J. (2007). Searching for Shereshevskii: What is superior about the memory of synaesthetes? Quarterly Journal of Experimental Psychology, 60, 681-695.

Yonelinas, A. P., Aly, M., Wang, W. C., \& Koen, J. (2010). Recollection and familiarity: Examining controversial assumptions and new directions. Hippocampus, 20, 1178-1194. 